Research Article

\title{
Complex Network Controllability Analysis on Business Architecture Optimization
}

\author{
Jicheng Chen $(\mathbb{D}$, Hongchang Chen, and Hanchao Li \\ National Digital Switching System Engineering and Technology Research Center, Information Engineering University, \\ Zhengzhou, Henan 450000, China
}

Correspondence should be addressed to Jicheng Chen; chenjicheng123@163.com

Received 4 February 2021; Accepted 18 March 2021; Published 28 March 2021

Academic Editor: Junming Huang

Copyright (C) 2021 Jicheng Chen et al. This is an open access article distributed under the Creative Commons Attribution License, which permits unrestricted use, distribution, and reproduction in any medium, provided the original work is properly cited.

Currently, the business process elements in the design of organizational business architecture are becoming increasingly complex, showing trends of networking and flattening. The control of complex business processes is a key factor for business architecture design. This paper considers a complex network generation method with business process and organization structure data and analyzes complex network controllability to examine the alignment of business processes and the organization structure. The uncontrollable nodes of the complex network can be identified, which act as the input for organization structure adjustment and business architecture optimization design.

\section{Introduction}

An organization's business architecture design refers to the planning and analysis procedure of business strategies, which determines the implementation mechanism of business strategies and supports business system development $[1,2]$. The business process description and organization structure design are two key parts of business architecture design: a business process is a collection of structured tasks or activities that can produce a particular service or product for one or more consumers [3]; an organizational structure is one way for companies to efficiently manage human-owned resources [4]. The alignment of business processes and organization structures has always been a key concern of business architecture design $[4,5]$.

The degree of support from an organizational structure for business processes is a key index for measuring the quality of the organizational structure [6-8]. An organization structure can be utilized to control business processes and drive them in an efficient way. Currently, with the increases in networking and the complexity of business processes, it is difficult for an organization structure to control business processes $[4,5]$. This paper introduces the term business process controllability, which refers to the adoption of fewer organizational nodes to achieve control of global business processes. Therefore, analyzing the control of organizational structures on business processes is an important indicator for business architecture design.

Complex network controllability (CNC), which emerged in the journal Nature in 2011 [9], bringing widespread attention to the subject [10-17], can be adopted to address the above question. The CNC refers to the control of an entire complex network with a few important nodes and determines whether the network is controllable [9]. In the development of business architecture, the combined network of business processes and organization nodes can be applied to excavate uncontrollable nodes: if the organizational nodes can control all the business processes, the business processes and organization structure are aligned. Additionally, the business architecture can be designed well with the above aligned business elements.

The rest of this paper is organized as follows. We first refine the research question on the basis of the theoretical background. Next, we propose an optimization design process for business architecture with the CNC method. This is followed by an illustrative case to explain the above process. Finally, we discuss our findings, as well as the limitations and implications of this paper. 


\section{Theoretical Foundation}

2.1. BPM in Business Architecture Design. Business process management (BPM) has persisted as one of the top-ranked concerns of business executives and IT executives [18, 19]. With the rapidly changing internal and external environments, the approach of adjusting and optimizing BPM determines organizational competitive advantages. The BPM process mainly includes process identification, process integration, process analysis, process redesign, process implementation, and process monitoring [19]. The alignment of business processes and organization structures is one of the key challenges in BPM according to Wang; organization structures and business processes have been subjected to management study for decades, and the relationship between them is a topic that has received only limited and fragmented theoretical treatment [20]. van der Aalst [19] pointed out that BPM mainly concerns five factors: process modeling languages, process enactment infrastructures, process model analysis, process mining, process flexibility, and process reuse. Among them, the authors deemed that establishing a good alignment between organizational structures and business processes can better achieve process flexibility and process reuse. vom Brocke and Sinnl [21] proposed 6 core elements of BPM: strategic alignment, government, methods, information technology, people, and culture. The strategic alignment aspect mainly includes the fitting of business processes and organizational structures. The authors also believe that the complexity of extant BPM is relatively high and that an efficient organizational structure can help control the complexity of business processes.

Business architecture is a key component of enterprise architecture design (e.g., application architecture and data architecture). Business architecture analysis helps sort out core business elements and their relationships in the organization and promotes the construction and evolution of business systems [1]. When considering BPM in business architecture design, it is beneficial to align business processes and the organization structure to obtain efficient business architecture solutions $[1,2]$. Vanhaverbeke and Torremans [5] argued that the adaptability degree of organizational units and business processes has a key role in optimizing organizations and processes. The existing business architecture design frameworks, such as the Department of Defense Architecture Framework 2.0 (DoDAF2.0) [22], Open Group Architecture Framework (TOGAF) [23], and Ministry of Defence Architectural Framework (MoDAF) [24], lack specific alignment methods and processes, although they include business activity entities, organization node entities, and relationship entities. Therefore, it is difficult for developers to design efficient business architectures and to guide practical applications.

Given the above explanations, Repa introduces a process normalization technique to build a process driven organization [6]; Hariawan describes how the organizational structure needs to support the business processes [4]; Fjeldstad and Snow explain how organization design is affected by value configuration and how new organization forms enable open and agile business models [7]. Overall, the extant research tends to analyze the relationships between business processes and organizational structures through subjective experience, practical discussion, or theoretical modeling and seldom conducts quantitative studies of organizational influences on business processes. The quantitative analysis of business process and organization structure alignment helps to more convincingly reorganize the structure.

2.2. CNC Research. The study of controllability is derived from classical cybernetics and represents that the system can be driven from any initial state to the desired state in limited steps. Starting from the ideas and methods of cybernetics, Liu et al. introduced the linear system structure controllability theory to study network structure controllability [9]. Through the introduction of graph matching theory, a CNC analysis framework that addresses the problem of the inapplicability of the traditional Kalman controllability matrix to large-scale complex networks is established [9].

Yuan introduced strict controllability to describe the controllability of arbitrary undirected and directed and weighted and unweighted complex networks [11]. Pósfai et al. investigated the influences of the clustering coefficient, community structure, and degree correlation coefficient on CNC [12]. Menichetti et al. studied the influences of the minimum in-degree nodes and out-degree nodes on controllability with a CNC analysis framework [13]. Hou et al. compared the controllability of the maximum entropy network and the BA network and found that the maximum entropy network has higher controllability than the BA network given the same conditions [14].

Jia et al. introduced a graph matching theory, proposed a $\mathrm{CNC}$ analysis framework based on the maximum matching method, and offered a minimum input theorem $[25,26]$. To address the maximum matching of a directed graph, the directed graph must first be transformed into a bipartite graph, which is explained in Figure $1 . V_{A}^{+}$and $V_{A}^{-}$are two point sets of the bipartite graph, where $V(A)^{+}=\left\{v_{1}^{+}, v_{2}^{+}, \ldots, v_{n}^{+}\right\}$and $V(A)^{-}=\left\{v_{1}^{-}, v_{2}^{-}, \ldots, v_{n}^{-}\right\}$. We assume that there is an edge pointing from node $i$ to node $j$ in $G(A)$ and that the corresponding directed edge in $\mathrm{H}(\mathrm{A})$ is pointing from $x_{i}$ to $x_{\mathrm{j}}$. According to the maximum matching algorithm, the maximum matching edge of $\mathrm{H}(\mathrm{A})$ can be calculated in $M(A)$. The node pointing from the largest matching edge is called a matching node, and the node not pointing from the largest matching edge is called a nonmatching node. The minimum input theorem has proven that the nonmatching nodes in the maximum matching network are uncontrollable nodes [25].

\section{Research Method}

With the above explanations, this paper mainly aims to align business processes and organizational structures with a CNC method, which helps obtain a better business architecture design solution. The research process is shown in Figure 2. 


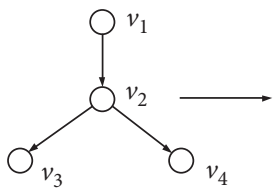

(a)

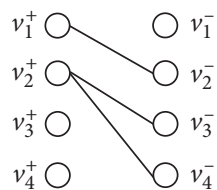

(b)

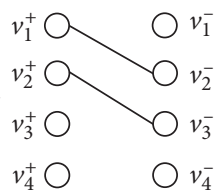

(c)

FIgURE 1: Two point sets of one bipartite graph. (a) G(A), (b) H(A), and (c) M(A).

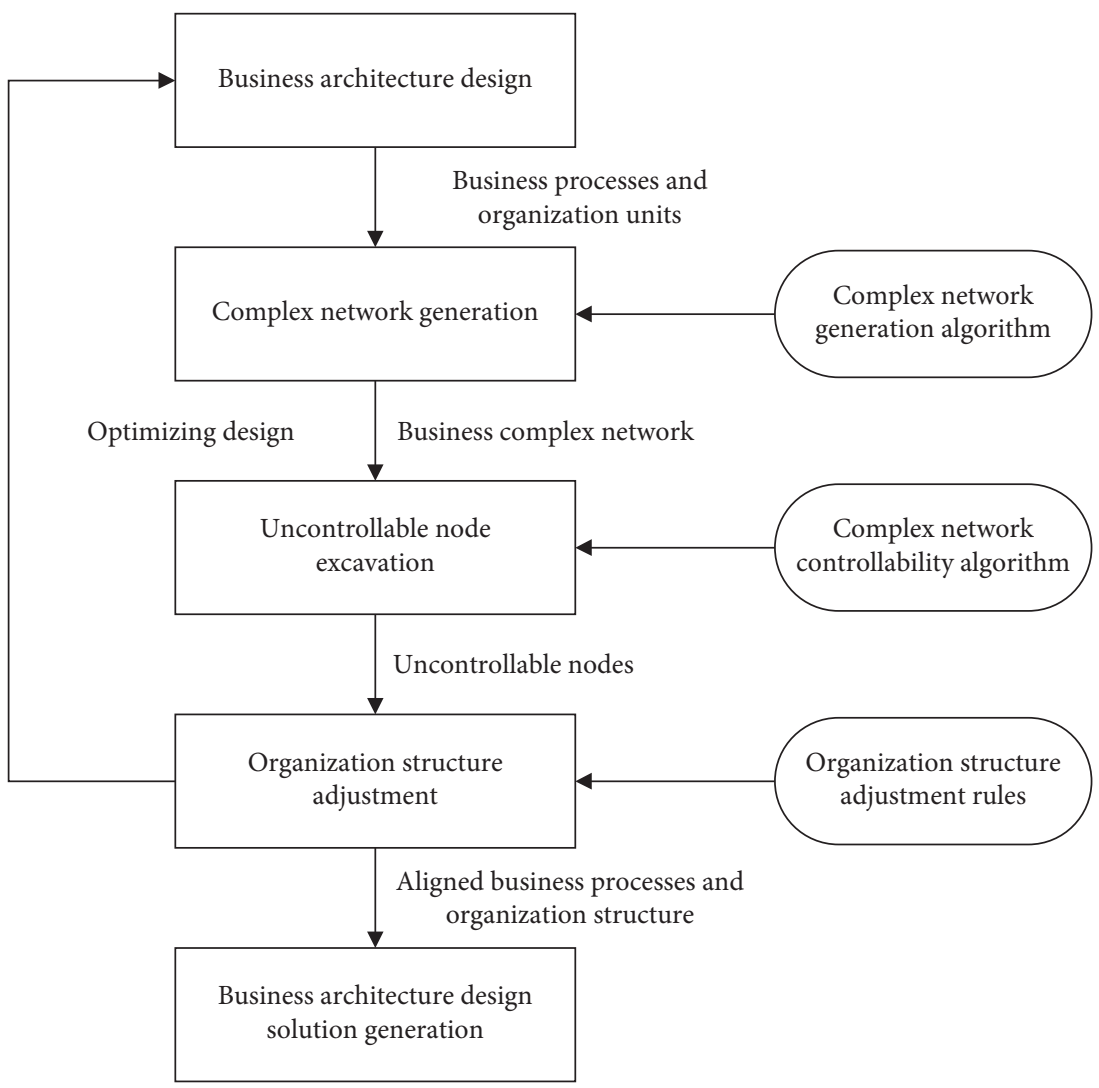

FIGURE 2: The alignment research process.

3.1. Business Architecture Design. The design process of business architecture mainly depends on the architecture frameworks, which often include business structure models, business activity models, organization structure models, business state models, etc. However, with regard to common frameworks such as TOGAF and DoDAF, the key developing elements are the business processes and organization structure. Business processes are often depicted visually in the form of a flowchart, which is displayed in Figure 3. Business processes are composed of business activities, which can convey business information and be decomposed into lower layers. For example, business activity B can transfer information to activity $\mathrm{C}$, and business activity $\mathrm{B}$ can be depicted by a child business process. In addition, the organization structure can be described by executing relationships from organization units to business activities, such as Org1 operating B11. The data generated in this step include business processes and their corresponding organization units, which can be used to analyze the controllability of the extant alignment solution in the following steps.
3.2. Complex Network Generation. The business processes, multitype business information, multiple layers, and organization units in the above can be used to produce complex business networks. First, we can extract one complex network from the business information among multiple layers. We assume that one business activity has the following attributes: InfoSourceSet refers to the node set in front of the information flows; InfoTargetSet refers to the node set at the end of the information flows; OISourceSet refers to the input node set; OITargetSet refers to the output node set; isLeaf refers to whether a node is a leaf node or not. The pseudocode is as follows.

Display all nodes in one page

(i) For all nodes

(ii) If node $2 \epsilon$ node 1 and node2. OISourceSet $=0$

(iii) node1.infoTargetSet add node2

(iv) If node $2 \epsilon$ node1 and node2. OITargetSet $=0$

(v) node1.infoSourceSet add node2 


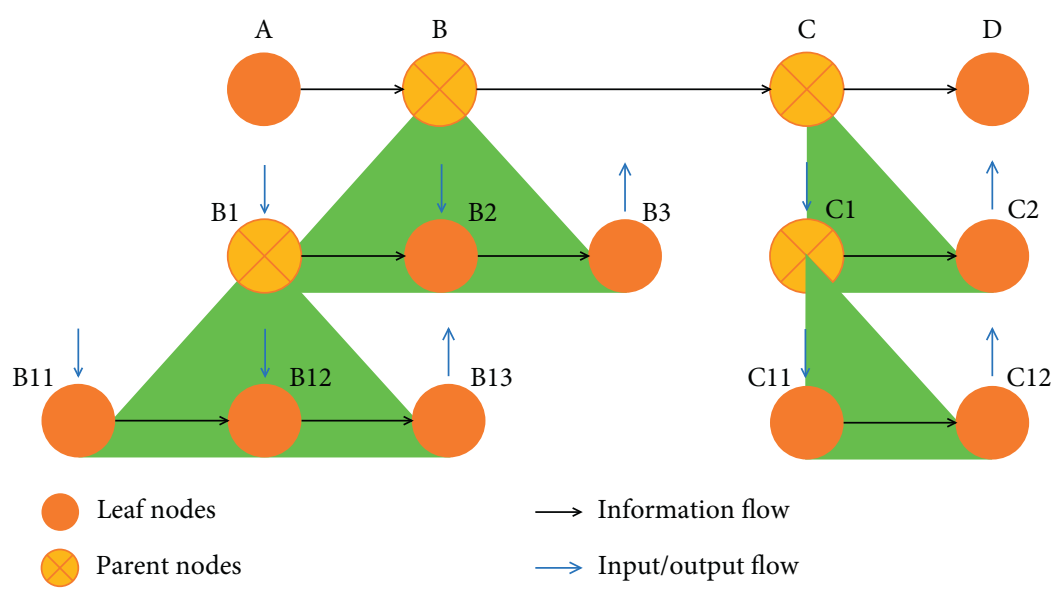

Figure 3: Business process design.

(vi) If node $2 \epsilon$ node1.InfoTargetSet and node2.isLeaf $=$ false

(vii) If node $3 \in$ node2.OITargetSet and node3.isLeaf $=$ true

(viii) node1.infoTargetSet add node3

(ix) node3.infoSourceSet add node1

(x) If node2 $\epsilon$ node1.OITargetSet and node $3 \epsilon$ node2.InfoTargetSet and node3.isLeaf $=$ false

(xi) If node4 $\epsilon$ node3.OITargetSet and node4.isLeaf $=$ true

(xii) node1.infoTargetSet add node4

(xiii) node4.infoSourceSet add node1

(xiv) Delete all redundant nodes and relations

Second, the connections between the organization units and corresponding business activities are added to the above complex network. A complex network combining the business processes and organization structure can thus be formed.

3.3. Uncontrollable Node Excavation. Uncontrollable nodes can be excavated by CNC algorithms. The CNC algorithm in this paper mainly adopts the methods of Liu et al. [9] and Jia et al. [25] to identify the maximum matching set and then classifies the controllable nodes and uncontrollable nodes. The main process is as follows.

Step 1. Identify redundant nodes and delete them from network G. The method is detailed in Reference [25].

Step 2. Obtain one maximum matching set in $G$ (denoted by $M)$.

Step 3. Randomly pick a node in $M$ (denoted by node i).

Step 4. Enumerate nodes in $M$ other than node $i$.

Step 5. Randomly pick another alternative maximum matching set as $M$.
Step 6. Repeat Step 3 until every maximum matching set has been excavated.

Step 7. Enumerate all controllable nodes and uncontrollable nodes [25].

3.4. Organization Structure Adjustment. The outcome of the above step can explain the alignment level of the business processes and organization structure. For example, if there are uncontrollable nodes, the extant organization structure cannot control the holistic business processes and should be adjusted. New organization units can be changed or added to adjust the organization structure. Several adjustment rules are as follows:

Rule 1: the minimum number of organization nodes to conduct input signals for business processes is adopted.

Rule 2: only one organization node for each workflow can adjust the organization nodes' execution location or delete redundant organization nodes.

Rule 3: organization nodes are added for uncontrolled workflows.

Ideally, the adjusted organization structure can control all the business processes. To examine the result, the above steps should be executed again.

3.5. Business Architecture Design Solution Generation. After better alignment of the business processes and organization structure is obtained, the other business architecture contents can be designed. Then, the whole business architecture design solution can be acquired, which is the basis for other organization architectures.

Overall, a good alignment of business processes and organizational structure can be obtained in the business architecture design stage with the above approach, which supports the optimization of the business architecture design.

3.6. Business Architecture Design. The business processes are developed with IDEF0 diagrams in this section. The main business process is shown in Figure 4, which includes the 


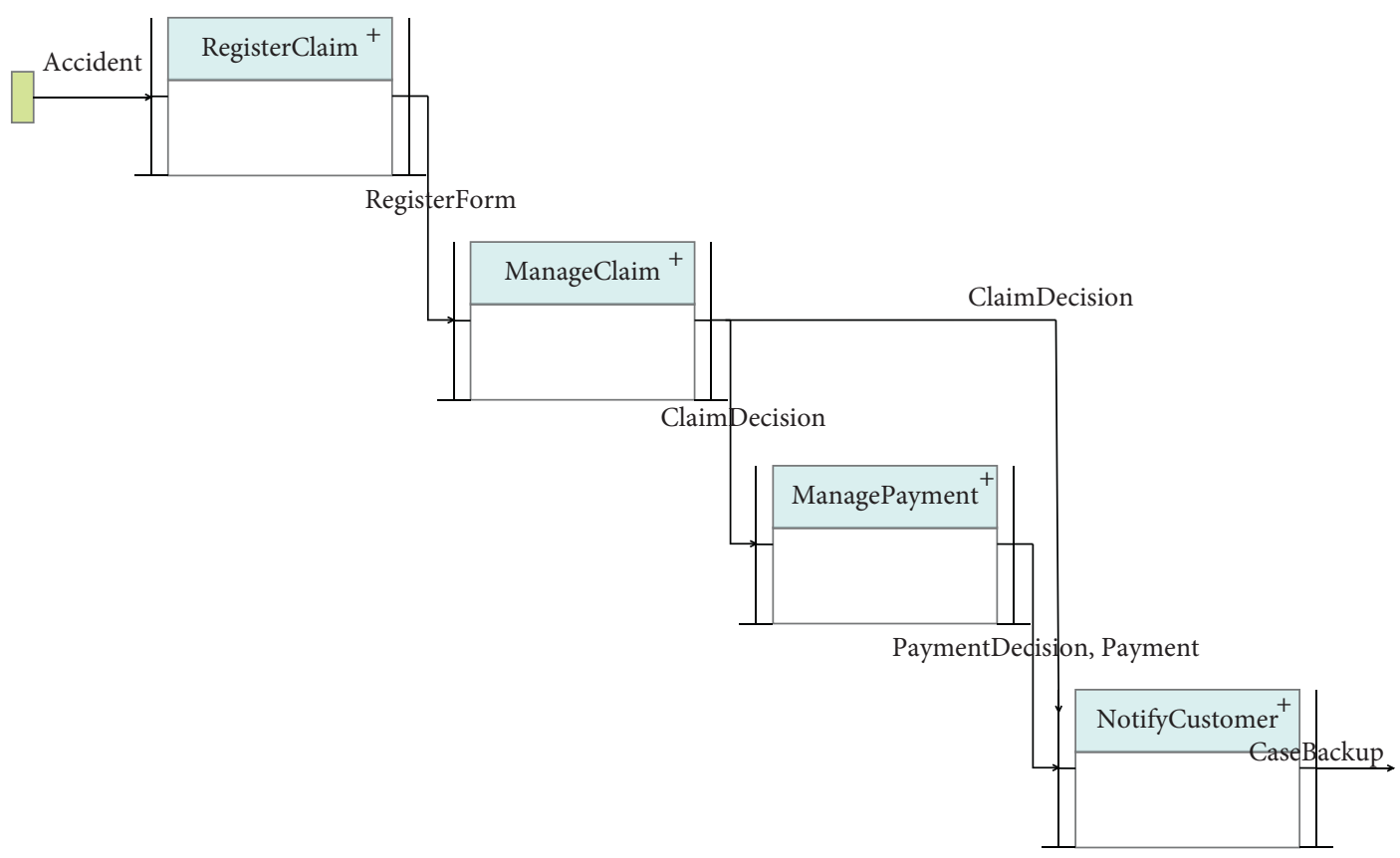

FIgURE 4: Top level business process.

RegisterClaim activity, ManageClaim activity, ManagePayment activity, and NotifyCustomer activity, like the ArchiSurance case. Multiple types of business information are transferred through business activities, such as RegisterForm and ClaimDecision. Each of the above activities can be decomposed to a lower layer. For example, the ManagePayment activity can be divided into the ReceiveDecision activity, ValueOrder activity, ReportClaim activity, and PayOrder activity. The lower layer process utilizes the upper layer information in the input and output interfaces and can also produce new specific information. This information can help form a complex network through the following steps. It is shown in Figure 5.

\section{An Illustrative Case Study}

To demonstrate the proposed process, we introduce the ArchiSurance case [1] in this paper. This case is often used in the business architecture community. As Iacob argued [1], this case is realistic and of manageable size without being overly simplistic. ArchiSurance is a fictitious organization that provides home, travel, and car insurance. It focuses on maintaining relationships with customers and handling claims and financial issues. Iacob developed the ArchiSurance architecture with the Archimate language [1]. For the sake of simplicity, we mainly develop the business process model in this paper. This model is explained according to the steps in Section 3.

4.1. Complex Network Generation. According to the above multilevel business processes, a complex business network can be generated, as shown in Figure 6. Due to having few business activities, the network is relatively simple. Given the ArchiSurance case, we assume that the organization unit FrontDesk can execute the workflow starting from the
ReceiveClaim activity; the organization unit BackDesk can execute the workflow starting from the ReceiveForm activity; and the organization unit Financial can execute the workflow starting from the ValueOrder activity. These organization units are displayed as input signals in Figure 6.

Uncontrollable Node Excavation. According to the algorithm in the above section, two uncontrollable nodes can be excavated from Figure 6: one is the ReceiveDecision node and the other is the ReportClaim node. The algorithm is achieved by means of the MATLAB toolset. These uncontrollable nodes expose the unaligned business processes and organization structure.

4.2. Organization Structure Adjustment. According to the algorithm idea, the ReceiveDecision node is uncontrollable because the signal of the JudgeClaim node is passed to the NotifyClaimDecision node; additionally, the ReportClaim node is uncontrollable because the signal of the ValueOrder node is passed to the PayOrder node. On the basis of the adjustment rules, since the workflow where the ReceiveDecision node is located is controlled by the BackDesk organization unit, the BackDesk organization unit can be changed to control the ReceiveDecision node. In addition, an exterior organization unit called the superior department can be added to the ReportClaim node as a control signal. In this way, we can control the above uncontrollable nodes. Reexamination of the CNC with the above algorithm shows that the new complex network is totally controlled.

4.3. Business Architecture Design Solution Generation. According to the adjusted organizational structure and its relationship with the business processes, the design of the 


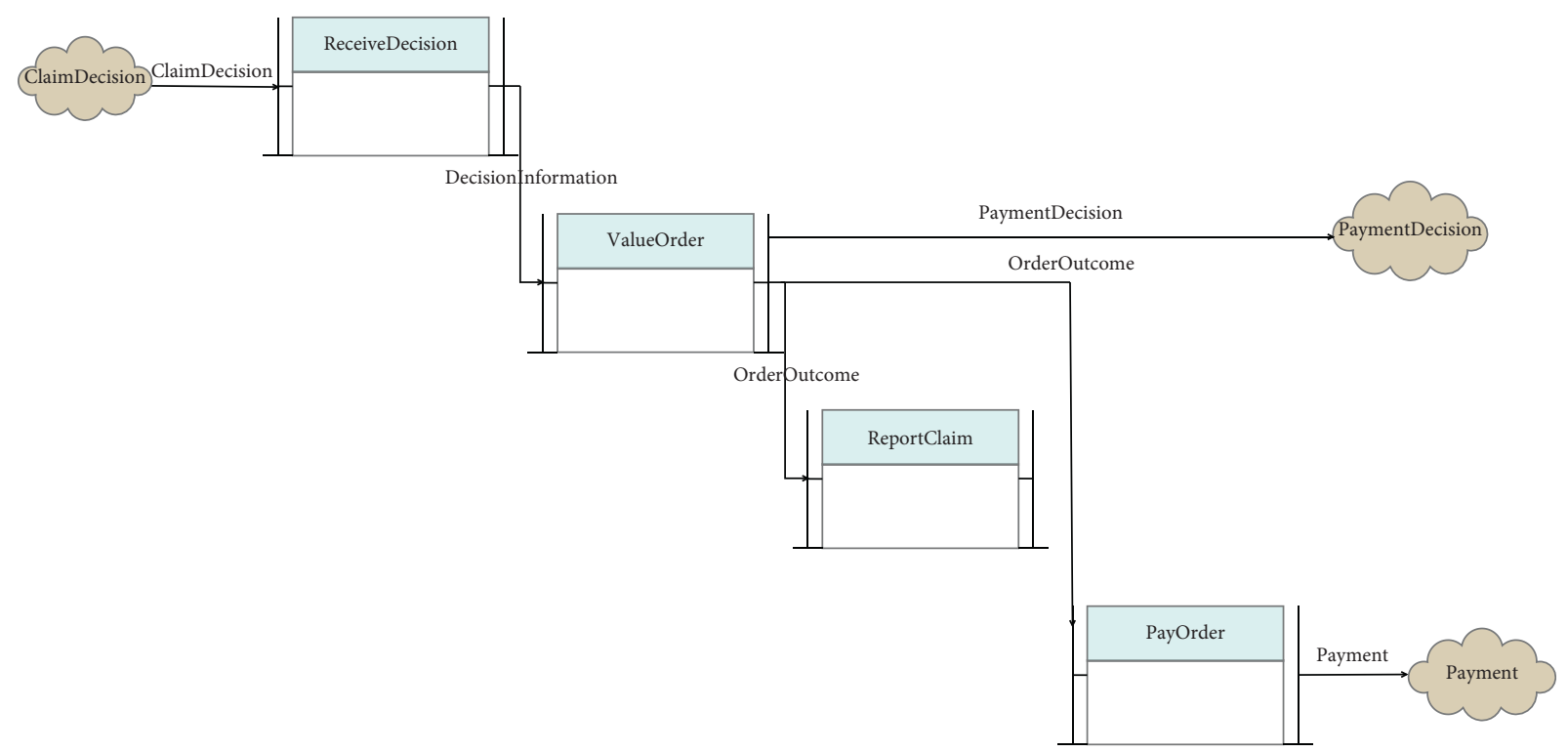

Figure 5: The lower level business process of the ManagePayment activity.

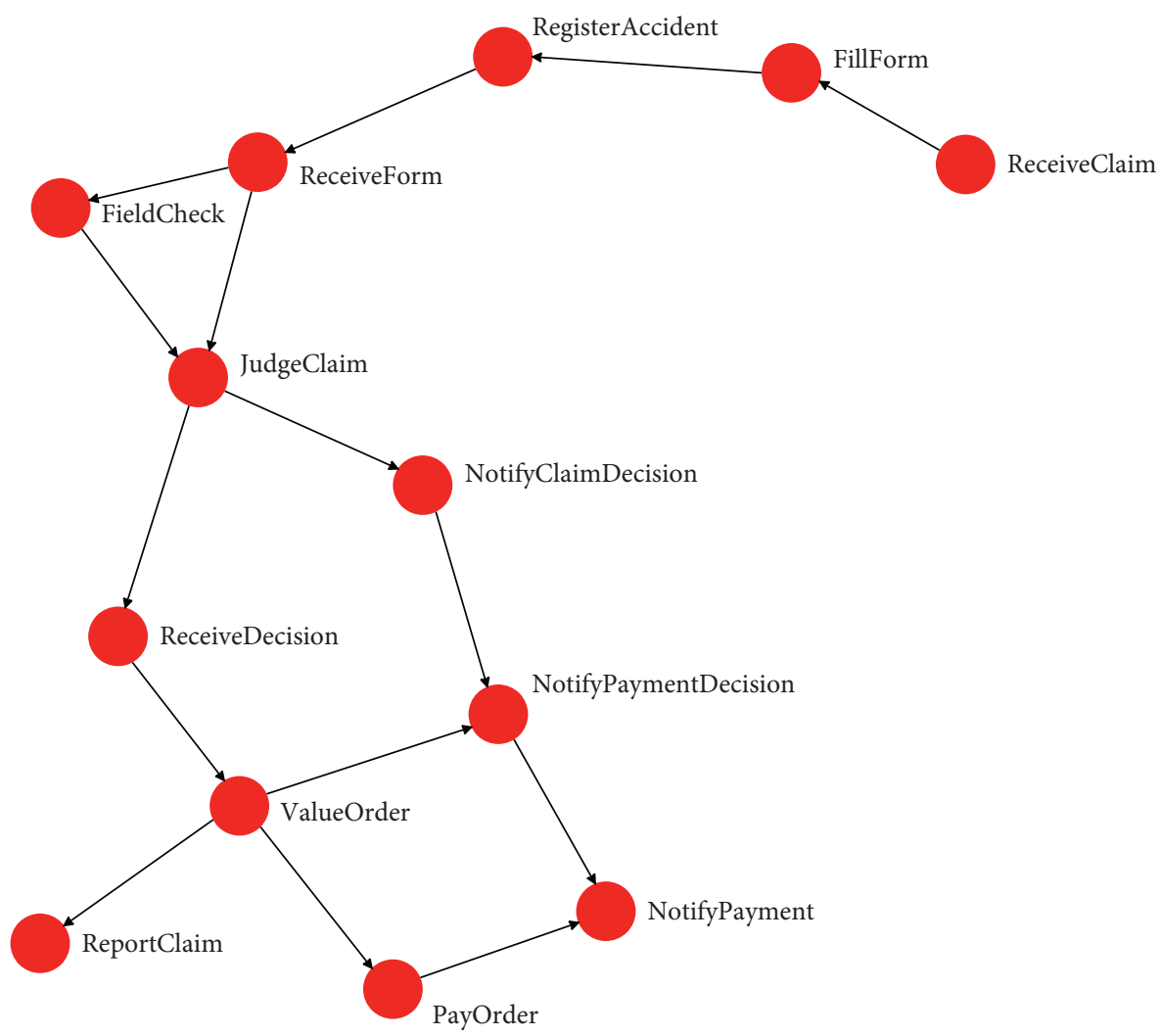

Figure 6: Business complex network.

business processes can be reorganized, and then other business architecture contents are developed. This paper does not explain this process in detail.

\section{Discussion and Conclusion}

Focusing on the alignment question of business processes and organizational structures in business architecture design, this paper considers the organization units' controllability and excavates the uncontrollable nodes from the business complex network to adjust the organization structure and optimize the business architecture design.

This paper provides insightful contributions compared to the literature. Theoretically, we consider the business process complexity and its fit with the organizational structure and analyze the business process controllability via 
a lens of complex network characteristics, which provides implications for future research. Practically, CNC analysis can reorganize organizational structures, which guides system adjustments.

This article still has limitations. First, the business process network generation considers only the amount of information flow and does not consider the types of information flow. Real business processes should contain multiple types of relationships. In addition, the case adopted in this article is illustrative and relatively simple, offering few network nodes. These limitations are motivation for further research. Further study should enrich the contents of the business processes and closely relate to actual situations. Furthermore, a practical case should be conducted to illustrate the $\mathrm{CNC}$ analysis and its importance on the alignment of business processes and organization structures.

\section{Data Availability}

No data were used to support this study.

\section{Conflicts of Interest}

The authors declare that they have no conflicts of interest.

\section{References}

[1] M. E. Iacob, L. O. Meertens, H. Jonkers et al., "From enterprise architecture to business models and back," Software and Systems Modeling, vol. 13, pp. 1059-1083, 2014.

[2] B. Fritscher and Y. Pigneur, "Business IT alignment from business model to enterprise architecture," International Conference on Advanced Information Systems Engineering, Springer Berlin Heidelberg, Berlin, Germany, 2011.

[3] R. K. L. Ko, S. S. G. Lee, and E. Wah Lee, "Business process management (BPM) standards: a survey," Business Process Management Journal, vol. 15, no. 5, pp. 744-791, 2009.

[4] F. Hariawan, "Organization Structure development based on company business process," Asian Journal of Innovation and Entrepreneurship, vol. 2, pp. 247-253, 2017.

[5] W. Vanhaverbeke and H. Torremans, "Organizational structure in process-based organizations," Knowledge and Process Management, vol. 6, no. 1, pp. 41-52, 1999.

[6] V. Repa, "Building a process driven organization with the process normalization technique," Complex Systems Informatics and Modeling Quarterly, vol. 14, pp. 22-37, 2018.

[7] Ø. D. Fjeldstad and C. C. Snow, "Business models and organization design," Long Range Planning, vol. 51, no. 1, pp. 32-39, 2018.

[8] P. Mathur and M. Nair, "Organization structure a key to driver to competitive advantage," International Journal of Management and Commerce Innovations, vol. 3, no. 2, pp. 348-356, 2016.

[9] Y.-Y. Liu, J.-J. Slotine, and A.-L. Barabási, "Controllability of complex networks,” Nature, vol. 473, no. 7346, pp. 167-173, 2011.

[10] X. F. Wang and G. Chen, "Pinning control of scale-free dynamical networks," Physica A: Statistical Mechanics and Its Applications, vol. 310, no. 3-4, p. 521, 2002.

[11] Z. Yuan, C. Zhao, Z. Di et al., "Exact controllability of complex networks," Nature Communications, vol. 4, p. 2447, 2013.
[12] M. Pósfai, Y.-Y. Liu, J.-J. Slotine, and A.-L. Barabási, "Effect of correlations on network controllability," Scientific Reports, vol. 3, pp. 1065-1067, 2013.

[13] G. Menichetti, L. Dall'Asta, and G. Bianconi, "Network Controllability Is Determined by the Density of Low InDegree and Out-Degree Nodes," Scientific Reports, vol. 113, Article ID 078701, 2014.

[14] M. Liang, S. Jin, D. Wang et al., "Optimization of controllability and robustness of complex networks by edge directionality," European Physical Journal B, vol. 89, p. 186, 2016.

[15] J. Zhao, J. Lu, and X. Wu, "Pinning control of general complex dynamical networks with optimization," Science China Information Sciences, vol. 53, no. 4, pp. 813-822, 2010.

[16] L. Hou, S. Lao, M. Small, and Y. Xiao, "Enhancing complex network controllability by minimum link direction reversal," Physics Letters A, vol. 379, pp. 1321-1325, 2015.

[17] Y. Xiao, S. Lao, L. Hou, M. Small, and L. Bai, "Effects of edge directions on the structural controllability of complex networks," PLoS One, vol. 10, no. 8, Article ID e0135282, 2015.

[18] M. Hammer, "What is business process management?" Handbook on Business Process Management, vol. 1, pp. 3-16, 2010.

[19] W.M. P. van der Aalst, "Business Process Management: A Comprehensive Survey," ISRN Software Engineering, vol. 37, Article ID 507984, 2013.

[20] S. Wang and X. Zhou, "New insights into organization structure and business process: an integrative point of view," Open Journal of Business and Management, vol. 07, no. 04, pp. 1953-1961, 2019.

[21] J. vom Brocke and T. Sinnl, "Culture in business process management: a literature review," Business Process Management Journal, vol. 17, no. 2, p. 357, 2011.

[22] The Open Group, TOGAF "Enterprise Edition" Version 8.1, The Open Group, San Francisco, CA, USA, 2003.

[23] U.S.: Department of Defense, DoD Architecture Framework Version 2.0, U.S.: Department of Defense, Virginia, USA, 2009.

[24] B. Biggs, Ministry of Defence Architectural Framework (Modaf), U.S.: Department of Defense, Virginia, USA, 2005.

[25] T. Jia, Y.-Y. Liu, E. Csóka, M. Pósfai, J.-J. Slotine, and A.-L. Barabási, "Emergence of bimodality in controlling complex networks," Nature Communications, vol. 4, pp. 1-6, 2002.

[26] S. Nie, X. Wang, B. Wang et al., "Effect of correlations on controllability transition in network control," Science Reports, vol. 6, p. 23952, 2016. 